\title{
Validity of the Neck Meridian Test as a Measure of Stress
}

\author{
Yasuhiro Honda ${ }^{1}$, Akira Tsuda ${ }^{2}$, Satoshi Horiuchi ${ }^{3,4}$ \\ ${ }^{1}$ Fukuoka Tenjin Medical Rehabilitation Academy, Fukuoka, Japan \\ ${ }^{2}$ Department of Psychology, Kurume University, Kurume, Japan \\ ${ }^{3}$ Health Sciences University of Hokkaido, Sapporo, Japan \\ ${ }^{4}$ Japan Society for the Promotion of Science, Kojimachi, Japan \\ Email: tomiyasuseikou2010seikouaiai@yahoo.co.jp
}

Received August 12, 2012; revised September 18, 2012; accepted October 7, 2012

\begin{abstract}
The neck meridian test is a procedure in which the participant is requested to stretch the neck in four directions and rate the intensity of pain felt and/or symptoms. The total score of the four responses has been shown to correlate with the level of perceived stress, and it has been suggested that it may be possible to use this test as a measure of perceived stress. The purpose of this study was to examine the effect of experimentally induced stress on the neck meridian test score. It was predicted that the neck meridian test score would increase only in participants who were exposed to stress manipulation. The participants were 19 male and 9 female college students (age, $34.1 \pm 9.37$ years) majoring in acupuncture and moxibustion medicine. The participants were randomly assigned to a stress group and a control group. All participants were requested to rest for 3 min and then complete the neck meridian test. Subsequently, they were administered a stress questionnaire. The participants in the stress group were instructed to prepare mentally for 3 min for a small 1-min examination that included performance in front of a judge, while those in the control group were requested to rest for additional $3 \mathrm{~min}$. After each period, the participants completed the neck meridian test and were administered a stress questionnaire. The stress score increased significantly only in the stress group, indicating that the experimental protocol was a valid means of inducing a stressed state. The neck meridian test score also increased only in the stress group, providing supporting evidence that the neck meridian test is a valid tool for assessing perceived stress. It is suggested that the test could be used in future studies applying techniques of acupuncture and moxibustion medicine to stress care.
\end{abstract}

Keywords: Neck Meridian Test; Perceived Stress; Acupuncture and Moxibustion Medicine

\section{Introduction}

Perceived stress inversely affects mental and physical health $[1,2]$. However, it is known that large number of people are stressed in many countries [3,4]. Thus, it is important to manage stress in order to prevent stressrelated health problems $[5,6]$.

Acupuncture and moxibustion represent treatment techniques in Chinese medicine. These methods depend to a large extent on the meridian theory, a representative theory of pathology. This theory assumes that energy (Qi) flows through meridians, which are invisible circuitries of channels in the body. If the energy flow is too slow, fast, turbulent, or static (Qi imbalance), mental and physical health is disturbed. Such Qi imbalance is caused by three main factors: 1 ) internal causes (emotional dis turbance); 2) external causes (environmental stressors); and 3) other causes (disturbances in life style, excessive work, and unhealthy sex). Acupuncture and moxibustion are used to correct Qi imbalance by stimulating the acu puncture points.
Acupuncture and moxibustion are popular in Japan. At least $1 \%-5 \%$ of Japanese people aged $>20$ years receive one of these treatments [7]. Interestingly, one fifth of such users tend to receive these treatments to reduce stress [7]. Thus, it may be useful to use acupuncture and moxibustion for stress management.

Unfortunately, a limited number of empirical studies have examined the effects of acupuncture and moxibus tion on stress in non-clinical human populations $[8,9]$. One of the obstacles to research in non-clinical human populations may be the lack of an objective means of assessing stress [10]. At present, researchers and practi tioners have to use psychological (e.g., questionnaires) and physiological measures (e.g., heart rate variability). The development of an objective and easy to use measure of perceived stress would provide a useful measurement tool for both researchers and practitioners who are inter ested in assessing stress.

Recently, Honda, Tsuda, \& Horiuchi [11] suggested the neck meridian test as a tool for measuring perceived 
stress. The neck meridian test is a procedure in which the participant is requested to stretch the neck in four directions and rate the intensity of pain felt and/or symptoms observed. The total score is then calculated. This test is a simplified version of the meridian test developed by Mukaino [12]. The meridian test is based on the assumption that Qi imbalance can be assessed as pain and/or symptoms associated with movements of the body. Honda et al. [11] found that the total test score was positively and significantly correlated with the stress score ( $\mathrm{r}$ $=0.40, \mathrm{p}<0.01)$. The test score was also reported to be affected by unhealthy behaviors such as drinking excessive alcohol or not eating a balanced diet. Since these unhealthy behavior habits seem not to change in a short period, this result suggests that the neck meridian test can be used as an indicator of perceived stress in research and practice in acupuncture and moxibustion.

The study of Honda et al. [11] was cross-sectional, and it is unclear whether an individual's neck meridian test score increases when the individual becomes stressed. A demonstration using a longitudinal research design that a change in the neck meridian test score corresponds to a change in the level of perceived stress would provide a more robust test of the validity of the neck meridian test as a measure of perceived stress.

Therefore, the purpose of this study was to examine the effect of experimentally induced stress on participants' scores on the neck meridian test. It was predicted that experimentally induced stress would increase the neck meridian test score. Demonstrating a significant increase of the neck meridian test score only in individuals who are stressed experimentally would provide more robust evidence supporting validity of the neck meridian test as an indicator of perceived stress.

\section{Method}

\subsection{Participants}

Eighteen male and 7 female college students participated in this study. Their mean age was 34.6 years, with a standard deviation of 9.69. After receiving an explanation of the purpose and protocol of the experiment, all participants gave written informed consent. The exclusion criteria were as follows: 1) current or previous physical or psychiatric illnesses and 2) receiving any medication. Unfortunately, because of a lack of available data, it was not possible to determine how representative the participants were as a sample of Japanese college students who major in acupuncture and moxibustion medicine.

\subsection{Experimental Manipulation}

The manipulation to induce a stressed state was to instruct each participant to anticipate a small examination.
The examination was 1-min long and required each student to perform several techniques in front of the first author. The outcome of this examination was partially relevant to the student's grade.

\subsection{Measures}

Subjective stress and activation were assessed in each session using an adaptation of the Japanese UWIST mood adjective checklist (JUMACL) [13]. This is based on the UWIST mood adjective checklist [14] and consists of 12 items that measure the fundamental dimensions of energetic arousal and tense arousal (TA). Each question was rated on a 4-point scale and scores were summed, so that higher energetic arousal and tension arousal values reflect greater energetic arousal and tension, respectively. The internal reliability and discriminant validity of the measure has been confirmed in Japanese college students [13]. Only the TA subscale was used in this study.

The neck meridian test was developed by Honda et al. [11]. Each participant was instructed to stretch the neck in four directions (neck extension, neck right rotation, neck left rotation, and neck flexion). Figure 1 shows the four movements in the neck meridian test. Then s/he was requested to rate how strong intensity of pain and/or symptoms s/he felt associated with each movement on a 4 -point scale $(0=$ not at all, $3=$ very strong). The total score was calculated. Reliability was evaluated in terms of internal consistency using Cronbach's alpha coeffi cient and was found to be acceptable $(\alpha=0.77)$ [11]. The validity of the measure was initially examined against a measure of stress. The neck meridian test score was significantly and positively related to the stress score measured with the tense arousal subscale of JUMACL $(r=$ 0.40) [11].

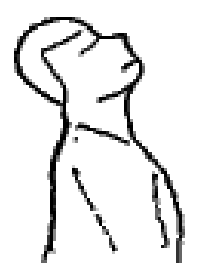

(a)

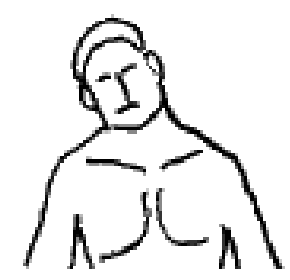

(c)

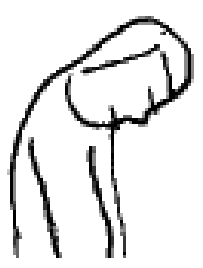

(b)

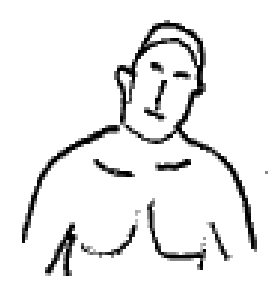

(d)
Figure 1. Movements of neck extension (a); Neck right rotation (b); Neck lateral right flexion (c); and Neck lateral left flexion (d). 


\subsection{Procedure}

The participants were randomly assigned to a stress group and a control group. All participants were requested to rest for $3 \mathrm{~min}$ and then complete the neck meridian test. After completing the test, they were administered JUMACL. The participants in the stress group were instructed to anticipate and prepare mentally for $3 \mathrm{~min}$ for a small 1-min examination, while those in the control group were requested to rest for additional $3 \mathrm{~min}$. After each period, the participants completed the neck meridian test and were administered JUMACL. No incentive was given to the participants.

\section{Results}

\subsection{Demographics}

First, we tested whether the two groups were matched with regard to the male/female ratio and mean age. These data are shown in Table 1. Chi-square test indicated that there was no significant difference in the male/female ratio $\left[\chi^{2}(1)=0.49, \mathrm{p}=0.48\right]$. Unpaired t-test found that the groups were fairly matched with regard to age $[\mathrm{t}(19)$ $=-0.27, \mathrm{p}=0.79]$.

\subsection{Validity of the Experimental Manipulation}

Second, we examined whether the manipulation would actually induce stress in participants in the stress group. The validity of the manipulation would be supported if a significant increase in the TA score was observed only in the stress group. Analysis of variance considering group (stress vs control) and period as independent variables revealed a significant group by period interaction $[\mathrm{F}(1$, $23)=24.42, p<0.01]$. Subsequent analyses indicated that the TA score increased significantly over time in the stress group and was significantly higher after the experiment $(\mathrm{p}<0.01)$ (Figure 2).

\subsection{Effect of Stress on the Neck Meridian Test Score}

Finally, we tested whether a change in the neck meridian test score would correspond to the change in the stress score. It was predicted that the neck meridian test score would increase over time only in the stress group. The

Table 1. Demographics.

\begin{tabular}{ccc}
\hline & Group & \\
\cline { 2 - 3 } & Stres group & Control group \\
\hline $\mathrm{N}$ & 12 & 13 \\
Male/female & $9 / 3$ & $9 / 4$ \\
Age & $34.5(8.68)$ & $34.7(11.45)$ \\
\hline
\end{tabular}

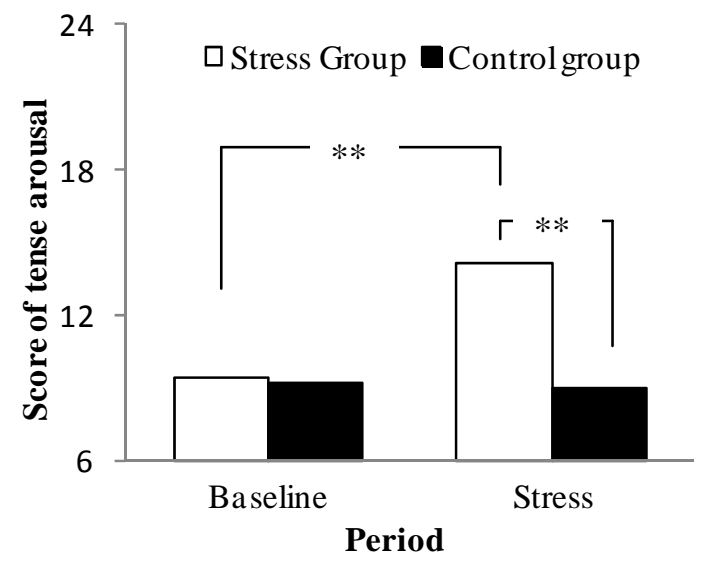

Figure 2. Changes in stress scores of the stress and control groups. ${ }^{* *} \mathrm{p}<\mathbf{0 . 0 1}$.



Figure 3. Changes in the scores on the neck meridian test for the stress and control groups. ${ }^{* *} \mathrm{p}<0.01$.

prediction would be supported if a significant increase in the score was observed only in the stress group. Analysis of variance considering group and period as independent variables revealed a significant group by period interacttion $[\mathrm{F}(1,23)=24.38, \mathrm{p}<0.01]$. Subsequent analyses indicated that the neck meridian test score increased significantly over time in the stress group, and it was significantly higher after the experiment (Figure 3).

\section{Discussion}

The purpose of this study was to examine the effect of experimentally induced stress on the neck meridian test score. Consistent with the prediction, the neck meridian test score increased in the group in which the participants were experimentally stressed. This result provides support for the validity of the test as a measure of perceived stress.

The anticipation of a small examination was valid as an experimental procedure to induce a stressed state. The TA score of JUMACL increased in the stress group, while no such change was observed in the control group. 
The TA subscale is a measure of perceived stress and has been found to increase during mental stress [14,15]. A significant increase in the TA score only in the stress group indicates that the experimental manipulation was valid as a means of inducing stress.

The present findings provide evidence that the neck meridian test can be used as a measure of perceived stress in the practice of acupuncture and moxibustion medicine. It is meaningful for practitioners to have such an option. Although stress is an important topic in clinical practice, no objective assessment tool had been available before the neck meridian test was suggested. For example, Honda, Tsuda, and Horiuchi [16] used a questionnaire (i.e., the Japanese version of the Rhode Island Stress and Coping Inventory [17]) to evaluate the effect of acupressure, a treatment method in acupuncture and moxibustion medicine, on perceived stress. Data obtained in this study implied that the neck meridian test can be used to measure a change in perceived stress in research and practice in acupuncture and moxibustion.

The findings also provide an alternative option for assessing stress. To date, stress has been measured mainly using psychological, physiological, sociological measures [18]. Very few body sensation-based measures of perceived stress have been developed. The neck meridian test attempts to assess perceived stress using bodily sensations (i.e., pain and symptoms) associated with stretching of the neck. Such a tool may be useful for individuals who are not good at recognizing and expressing their own feelings. Thus, the neck meridian test is a potential alternative to current stress measurement tools.

Although this study provides evidence to support the validity of the neck meridian test as a measure of perceived stress, some limitations should be addressed. The main limitation of this study was that the participants were college students who were studying acupuncture medicine. We need to extend our findings to other college students and populations. This extension would be very important because our participants were expected to have more extensive knowledge of the acupuncture points compared with college students majoring in other subjects. Furthermore, it is necessary to replicate our findings in populations other than college students. Another limitation is that stress was measured using only a single questionnaire. It is necessary to assess stress with other questionnaires such as the Depression Anxiety Stress Scales [19] and more objective measures such as sympathetic nervous activity and cortisol levels.

\section{Acknowledgements}

This work was supported by Grant-in-Aid for Scientific Research (B) (22330196) to AT. The authors would like to thank Enago (www.enago.jp) for the English language review.

\section{REFERENCES}

[1] M. S. Kopp, Á. Skrabski, A. Székely, A. Stauder and R. Williams, "Chronic Stress and Social Changes, Socioeconomic Determination of Chronic Stress," Annals of the New York Academy of Sciences, Vol. 1113, 2007, pp. 325-338. doi:10.1196/annals.1391.006

[2] K. Arabia, I. Abdelrahim and I. Humaida, "Relationship between Stress and Psychosomatic Complaints among Nurses in Tabarjal Hospital,” Open Journal of Medical Psychology, Vol. 1 No. 3, 2012, pp. 15-19. doi:10.4236/ojmp.2012.13003

[3] N. B. Anderson, K. C. Nordal, S. J. Breckler, D. Ballard, L. Bufka, L. Bossolo, et al., "Stress in America Findings,” American Psychological Association, 2010. http://www.apa.org/news/press/releases/stress/national-re port.pdf

[4] European Foundation for the Improvement of Living and Working Conditions, "Fourth European Working Conditions Survey," Office for Official Publications of the European Communities, Luxembourg, 2007.

http://www.eurofound.europa.eu/pubdocs/2006/98/en/2/ef 0698en.pdf

[5] S. Horiuchi, A. Tsuda, E. Kim, K.-S. Hong, Y.-S. Park and U. Kim, "Relationships between Stage of Change for Stress Management Behavior and Perceived Stress and Coping,” Japanese Psychological Research, Vol. 52, No. 4, 2010, pp. 291-297. doi:10.1111/j.1468-5884.2010.00444.x

[6] S. Horiuchi, A. Tsuda, H. Kobayashi and J. M. Prochaska, "The Reliability and Validity of the Japanese Version of Pro-Change's Decisional Balance Measure for Effective Stress Management (PDSM)," Japanese Psychological Research, Vol. 54, No. 2, 2012, pp. 128-136. doi:10.1111/j.1468-5884.2011.00490.x

[7] T. Yano, N. Ishizaki and K. Kawakita, "Survey on the Acupuncture and Moxibustion Therapy-Focused on the Patients' Behavior," Annual Report of the Foundation for Training and Licensure Examination in Anma-MassageAcupressure, Acupuncture and Moxibustion, 2005.

[8] F. Fukuda, "The Stress Management and Acupuncture and Moxibustin Medicine," Japanese Journal of Stress Science, Vol. 23, No. 1, 2008, pp. 82-94.

[9] N. Robinson, A. Lorenc and X. Liao, "The Evidence for Shiatsu: A Systematic Review of Shiatsu and Acupressure," BMC Complementary and Alternative Medicine, Vol. 11, 2011, p. 88.

[10] Y. Honda, A. Tsuda and S. Horiuchi, "Relationships between Positive Responses Associated with M-Test and Perceived Stress," Oriental Medicine and Pain Clinic, Vol. 42, No. 1-2, 2012, pp. 17-25.

[11] Y. Honda, A. Tsuda and S. Horiuchi, "The Neck Meridian Test as an Assessment Tool of Perceived Stress," Oriental Medicine and Pain Clinic, in Press.

[12] Y. Mukaino, "Sports Acupuncture: The Meridian Test and Its Application,” East Land Press, Seattle, 2008.

[13] G. Matthews, D. M. Jones and A. G. Chamberlain, "Re- 
fining the Measurement of Mood: The UWIST Mood Adjective Checklist,” British Journal of Psychology, Vol. 81, No. 1, 1990, pp. 17-42. doi:10.1111/j.2044-8295.1990.tb02343.x

[14] H. Okamura, A. Tsuda, J. Yajima, H. Mark, S. Horiuchi, N. Toyoshima and T. Matsuishi, "Short Sleeping Time and Psychobiological Responses to Acute Stress," International Journal of Psychophysiology, Vol. 78, No. 3, 2010, pp. 209-214. doi:10.1016/j.ijpsycho.2010.07.010

[15] S. Horiuchi, A. Tsuda, H. Okamura, J. Yajima and A. Stepoe, "Differential Elicitation of the Salivary 3-Methoxy-4-Hydroxyphenylglycol (MHPG) Responses by Mental Stress Testing," Japanese Journal of Behavioral Medicine, Vol. 16, No. 1, 2010, pp. 31-38.

[16] Y. Honda, A. Tsuda and S. Horiuchi, "Effect of a Four-
Week Self-Administered Acupressure Intervention on Perceived Stress over the Past Month,” Open Journal of Medical Psychology, Vol. 1 No. 3, 2012, pp. 20-24. doi:10.4236/ojmp.2012.13004

[17] S. Horiuchi, A. Tsuda, Y. Tanaka, J. Yajima and S. Tsuda, "Development of the Japanese Version of the Rhode Island Stress and Coping Inventory: Examination of Reliability and Validity," Behavioral Science Research, Vol. 47, No. 1, 2008, pp. 37-46.

[18] S. Cohen, R. C. Kessler and U. L. Gordon, "Measuring Stress: A Guide for Health and Social Scientists,” Oxford Press, New York, 1995.

[19] S. H. Lovibond and P. F. Lovibond, "Manual for the Depression Anxiety Stress Scales,” 2nd Edition, Psychology Foundation, Sydney, 1995. 